\title{
ECOCARDIOGRAFIA NA HIPERTENSÃO PULMONAR ESQUISTOSSOMÓTICA
}

\author{
Antônio Emanuel1, Francisco de Assis², Vera Lucato', Aluizio Prata1, \\ Evandro Osterne ${ }^{2}$ e Osório Luiz Rangel de Almeida2.
}

\begin{abstract}
Realizou-se estudo ecocardiográfico em 16 pacientes esquistossomóticos, 11 dos quais tinham hipertensão pulmonar comprovada por cateterismo cardíaco. Em sete casos havia hipertensão pulmonar ao ecocardiograma, embora em um deles os níveis de pressão fossem normais.
\end{abstract}

Palavras chaves: Ecocardiografia. Hipertensão pulmonar esquistossomótica.

A ultra-sonografia passou a ser empregada em cardiologia no início da década de 50. O advento da ecocardiografia bidimensional elevou a sensibilidade $e$ fidedignidade do método, em virtude de sua maior precisão técnica ${ }^{1}$. Em principio, por questões técnicas, só foi utilizada para avaliação das válvulas mitral, tricúspide e aórtica. Gramiak e cols ${ }^{3}$ desenvolveram método para detectar a válvula pulmonar, e Nanda e cols 789 estudaram pacientes com hipertensão pulmonar e preconizaram parâmetros para o seu diagnóstico. Posteriormente o método foi utilizado por outros investigadores 512 , mas desconhecemos a sua aplicação em pacientes com hipertensão pulmonar esquistossomótica.

Neste trabalho preliminar apresentaremos os resultados obtidos em 16 pacientes esquistossomóticos, onze dos quais com hipertensāo pulmonar comprovada por cateterismo cardiaco.

\section{MATERIAL E MÉTODOS}

Os 16 pacientes esquistossomóticos procediam do Estado da Bahia, sendo sete do sexo masculino e nove do sexo feminino. A idade variou de 19 a 55 anos, com média de 32 anos (Tabela 1). Todos tinham ovos de Schistosoma mansoni nas fezes. As formas clinicas foram diagnosticadas como hepatointestinal e hepatoesplênica (Tabela 1), sendo o critério desta estabelecido de acordo com os parâmetros adotados por Prata ${ }^{10}$. O diagnóstico de hipertensão pulmonar esquistossomótica baseou-se numa pressão média $\geq$ $20 \mathrm{mmHg}$ na artéria pulmonar, conforme preconizado por Guimarāes ${ }^{4}$, e na ausência de outras possibilidades clínicas que cursam com hipertensão pulmonar.

1. Núcleo de Medicina Tropical e Nutrição/Universidade de Brasilia. Brasília, DF.

2. Hospital das Forças Armadas - Brasilia, DF.

Recebido para publicação em $23 / 1 / 87$.
Tabela 1 - Idade, sexo e forma clínica de 16 pacientes esquistossomóticos avaliados pela ecocardiografia.

\begin{tabular}{cccl}
\hline No do caso & $\begin{array}{c}\text { Idade } \\
\text { (anos) }\end{array}$ & Sexo & $\begin{array}{l}\text { Forma } \\
\text { Clínica }\end{array}$ \\
\hline 01 & 33 & $\mathrm{~F}$ & $\mathrm{HI}$ \\
02 & 34 & $\mathrm{~F}$ & $\mathrm{HE}$ \\
03 & 34 & $\mathrm{~F}$ & $\mathrm{HE}$ \\
04 & 21 & $\mathrm{M}$ & $\mathrm{HE}^{*}$ \\
05 & 26 & $\mathrm{~F}$ & $\mathrm{HE}^{*}$ \\
06 & 29 & $\mathrm{~F}$ & $\mathrm{HE}^{*}$ \\
07 & 19 & $\mathrm{~F}$ & $\mathrm{HE}$ \\
08 & 22 & $\mathrm{M}$ & $\mathrm{HE}$ \\
09 & 51 & $\mathrm{~F}$ & $\mathrm{HE}$ \\
10 & 24 & $\mathrm{M}$ & $\mathrm{HI}$ \\
11 & 38 & $\mathrm{~F}$ & $\mathrm{HE}$ \\
12 & 42 & $\mathrm{M}$ & $\mathrm{HE}^{*}$ \\
13 & 26 & $\mathrm{M}$ & $\mathrm{HI}$ \\
14 & 55 & $\mathrm{M}$ & $\mathrm{HE}^{*}$ \\
15 & 54 & $\mathrm{~F}$ & $\mathrm{HE}^{*}$ \\
16 & 23 & $\mathrm{M}$ & $\mathrm{HE}^{*}$ \\
\hline
\end{tabular}

HI - Hepatointestinal

HE - Hepatoesplênico

*-Esplenectomizado

Dos 16 pacientes que realizaram ecocardiografia, 14 o fizeram em ecocardiógrafo unidimensional, módulo M, marca Funbec, com transdutores de 2,5 e $5 \mathrm{MHz}$, e dois num ecocardiógrafo bidimensional, marca Sonel (CGR) setorial mecânico $\left(82^{\circ}\right)$. Consideramos que um paciente apresentava sinais ecocardiográficos sugestivos de hipertensão pulmonar quando, na impossibilidade de ser estudada a válvula pulmonar, havia aumento do diâmetro do ventrículo 
Emanuel A, Assis F, Lucato V, Prata A, Osterne E, Almeida OLR. Ecocardiografia na hipertensão pulmonar esquistossomótica. Revista da Sociedade Brasileira de Medicina Tropical 20: 115-117, Abr-Jun, 1987.

direito e de sua via de saida e que os sinais eram indicativos quando preenchiam os parâmetros preconizados por Nanda e cols. ${ }^{79}$ e Weyman e cols. ${ }^{12}$.

\section{RESULTADOS}

Dos 16 pacientes estudados (Tabela 2), onze $(68,7 \%)$ tinham hipertensão pulmonar ao cateterismo cardíaco (n.s 3, 4, 5, 7, 8, 9, 10, 11, 14, 15 e 16); em quatro não se registrou onda " $A$ " ao ecocardiograma por dificuldades técnicas; e nos 12 casos em que ela foi assinalada, em cinco era igual ou menor que $2 \mathrm{~mm}$ (n.s que o aumento do diâmetro do ventrículo direito e de sua via de saída era sugestivo do diagnóstico, só um deles (no 14) tinha hipertensão pulmonar de fato.

\section{COMENTÁRIOS}

Até o momento o diagnóstico preciso de hipertensão pulmonar continua sendo feito, exclusivamente, através do cateterismo cardíaco. Apesar de ser o único método que assegura o diagnóstico, não se pode deixar de considerar os perigos que envolvem o exame ${ }^{211}$. Assim, seria útil se dispuséssemos de um método não invasivo capaz de diagnosticar esses casos

Tabela 2 - Datos de cateterismo cardíaco e ecocardiografia de 16 pacientes esquistossomóticos

\begin{tabular}{|c|c|c|c|c|c|c|c|}
\hline \multirow[b]{2}{*}{$\begin{array}{l}\text { No do } \\
\text { Caso }\end{array}$} & \multicolumn{2}{|c|}{ Cateterismo Cardiaco } & \multicolumn{5}{|c|}{ Ecocardiografia } \\
\hline & $\begin{array}{c}\text { Pressão } \\
\text { Básica }\end{array}$ & $\begin{array}{l}\text { Pressão } \\
\text { Média }\end{array}$ & $\begin{array}{l}\text { Velocidade } \\
\qquad E F\end{array}$ & $\begin{array}{l}\text { Velocidade } \\
\qquad B C\end{array}$ & Onda " $A$ " & $\begin{array}{l}\text { Diâmetro de VD/ } \\
\text { Via de saida VD }\end{array}$ & $\begin{array}{c}\text { Conclusão } \\
(H P)\end{array}$ \\
\hline 01 & $28 / 8$ & 15 & NR & NR & NR & $19 / 28 \mathrm{~mm}$ & Não \\
\hline 02 & $28 / 13$ & 19 & NR & & NR & $/ 24 \mathrm{~mm}$ & Não \\
\hline 03 & $30 / 13$ & 20 & 40 & & $4 \mathrm{~mm}$ & $15 / 27 \mathrm{~mm}$ & Não \\
\hline 04 & $52 / 20$ & 31 & 26 & & $2 \mathrm{~mm}$ & $32 / 32 \mathrm{~mm}$ & $\mathrm{Sim}$ \\
\hline 05 & $45 / 18$ & 31 & 20 & 270 & $3 \mathrm{~mm}$ & $26 / 30 \mathrm{~mm}$ & Não \\
\hline 06 & $30 / 12$ & 18 & 0 & 300 & $5 \mathrm{~mm}$ & $/ 29 \mathrm{~mm}$ & Sim \\
\hline 07 & $28 / 14$ & 20 & 8 & & $4 \mathrm{~mm}$ & $20 / 29 \mathrm{~mm}$ & Não \\
\hline 08 & $32 / 18$ & 23 & 10 & & $5 \mathrm{~mm}$ & $25 / \mathrm{mm}$ & Não \\
\hline 09 & $55 / 24$ & 35 & Retificada & & $<2 \mathrm{~mm}$ & $20 / 25 \mathrm{~mm}$ & Sim \\
\hline 10 & $32 / 12$ & 20 & 0 & 260 & $0 \mathrm{~mm}$ & $25 / 30 \mathrm{~mm}$ & Sim \\
\hline 11 & $67 / 28$ & 41 & 5 & & $<2 \mathrm{~mm}$ & & Sim \\
\hline 12 & $30 / 10$ & 17 & NR & NR & NR & $32 / 25 \mathrm{~mm}$ & Sug \\
\hline 13 & $30 / 12$ & 18 & 18 & & $4 \mathrm{~mm}$ & $26 / 26 \mathrm{~mm}$ & Não \\
\hline 14 & $70 / 30$ & 48 & NR & NR & NR & $28 / 31 \mathrm{~mm}$ & Sug \\
\hline 15 & $55 / 22$ & 35 & Retificada & & $1 \mathrm{~mm}$ & $29 / 22 \mathrm{~mm}$ & Sim \\
\hline 16 & $42 / 15$ & 25 & 23 & & $2,5 \mathrm{~mm}$ & $25 / 34 \mathrm{~mm}$ & Sim \\
\hline
\end{tabular}

HP - Hipertensão pulmonar; Sug - Sugestivo; NR - Não registrado

$4,9,10,11$ e 15 ), em um (n. 5) era de $3 \mathrm{~mm}$, e em outro (no 16) 2,5mm, em três (nos 3, 7 e 13) de $4 \mathrm{~mm}$ e em dois (n.s 6 e 8 ) de $5 \mathrm{~mm}$. A velocidade EF não foi determinada em quatro casos, em três (n:s 6, 10 e 11) era menor que $6 \mathrm{~mm}$, em dois retificada e nos demais variou de 8 a $40 \mathrm{~mm} / \mathrm{seg}$. O diâmetro do ventriculo direito foi determinado em 13 casos e em nove ultrapassou $20 \mathrm{~mm}$. A via de saída do ventrículo direito foi medida em 14 casos, verificando-se que em todos ultrapassava $20 \mathrm{~mm}$. Assim, em 7 (43,7\%) pacientes (n.s 4, 6, 9, 10, 11, 15 e 16) os dados ecocardiográficos preenchiam os critérios para o diagnóstico de hipertensão pulmonar, embora um deles (n: 6) não a apresentasse. Dos dois (12,5\%) casos (nos 12 e 14) em com segurança. Sendo a ecocardiografia um desses métodos e considerando-se ser de grande eficácia no diagnóstico de hipertensão pulmonar, segundo Nanda e cols $^{7}$ e Weyman e cols ${ }^{12}$, ela poderia ser valiosa na determinação da hipertensão pulmonar esquistossomótica. Na nossa experiência preliminar, entretanto, a ecocardiografia documentou hipertensão pulmonar apenas em $54 \%$ dos pacientes diagnosticados pelo cateterismo cardiaco. Desse modo, $46 \%$ dos pacientes não teriam sido considerados portadores de hipertensão pulmonar caso a avaliação tivesse consistido apenas no exame ecocardiográfico. Por outro lado, em dois pacientes o estudo ecocardiográfico indicava ou sugeria a existência de hipertensão pulmonar e o cate- 
terismo cardiaco não a confirmou. Assim, a ecocardiografia unidimensional se mostrou um método de pouca sensibilidade no diagnóstico de hipertensão pulmonar esquistossomótica. Vale ressaltar que a maioria dos casos em que a ecocardiografia não evidenciou sinais de hipertensão pulmonar eram pacientes com elevação discreta dos niveis tensionais na artéria pulmonar. Talvez isso justifique a diferença das nossas observações com as de Nanda e cols ${ }^{7} \mathrm{e}$ Weyman e cols ${ }^{12}$. Nos que tinham hipertensão moderada observamos melhores resultados: apenas um deles não apresentava sinais ecocardiográficos indicativos. Como nas áreas endêmicas a maioria dos pacientes com hipertensão pulmonar esquistossomótica tem aumento leve a moderado dos niveis tensionais, a ecocardiografia unidimensional não nos parece um bom meio para o diagnóstico desses pacientes. Nos dois casos em que foi realizada a ecocardiografia bidimensional havia sinais de hipertensão pulmonar: um tinha hipertensão leve e outro, moderada. Como ela tem maior sensibilidade que a ecocardiografia unidimensional, é possivel que diagnostique melhor a hipertensão pulmonar esquistossomótica leve a moderada. Seria, então, conveniente o seu emprego nesses pacientes, inclusive em áreas endêmicas.

\section{SUMMARY}

An echocardiographic study was done in sixteen patients with schistosomiasis, eleven of whom had pulmonary hypertension proved by cardiac catheterization. In seven patients there was evidence of pulmonary hypertension on the echocardiogram although in one of these normal pressure levels were recorded.

Key words: Echocardiography. Schistosomal pulmonary hypertension.

\section{REFERÊNCIAS BIBLIOGRÁFICAS}

1. Feigenbaum H. Echocardiography. In: Wyngaarden \&
Smith (eds.). Cecil Textbook of Medicine. Philadelphia WB Saunders Company, 16? ed., pp. 118-122, 1982.

2. Franch RH. Cardiac catheterization. In: Hurst (ed.) The Heart Arteries and Veins. Londres, Mc Grow-Hill Kogakuska, 3ㄹ ed. cap. 23, 1974.

3. Gramiak R, Nanda NC, Shah FM. Echocardiographic detection of the pulmonary valve. Radiology 102: 153157, 1972.

4. Guimarães AC. Situação atual dos conhecimentos sobre o envolvimento cardiopulmonar na esquistossomose mansônica. Arquivos Brasileiros de Cardiologia 38: 301-309, 1982.

5. Haertel JC, Vitola D, Goldim JR, Azevedo DF, Zielinsky P. Análise critica das medidas obtidas através da ecocardiografia unidimensional para deteç̧ão e graduação da hipertensão arterial pulmonar. Arquivos Brasileiros de Cardiologia 45: 329-334, 1985.

6. Morcef FAP. Ecocardiografia. Guanabara Koogan, Rio de Janeiro, 1980.

7. Nanda NC, Gramiak R. Echocardiographic evaluation of pulmonary hypertension. Circulation 50: 575-581, 1974.

8. Nanda NC, Gramiak R, Robinson T, Shah PM. Evaluation of pulmonary hypertension by echocardiography. J. Clin. Ultrasound. 1: 255, 1973. In: Nanda NC, Gramiak R. Echocardiographic Evaluation of Pulmonary Hypertension. Circulation 50:575-581, 1974.

9. Nanda NC, Gramiak R, Shah PM, Robinson T. Echocardiographic diagnosis of pulmonary hypertension. Excerpta Medica 277-12, 1973. In: Nanda NC, Gramiak R. Echocardiographic Evaluation of Pulmonary Hypertension. Circulation 50: 575-581, 1974.

10. Prata A. II Simpósio sobre Esquistossomose. Organizado por A. Prata e Ernani Aboim. p. 1979, 1970.

11. Schafer H, Blain JM, Ceballos R, Bing RJ. Essentialpulmonary hypertension: a report of clinical-physiologic studies in three patients with death following catheterization of the heart. Ann. Inst. Med. 44: 505-523, 1956.

12. Weyman AE, Dilon JC, Feigenbaum H, Chang $\mathrm{S}$. Echocardiographic patterns of pulmonic valve motion with pulmonary hypertension. Circulation 50: 905-910, 1974. 